Pacific Journal of Mathematic 


\title{
GEOMETRIC THEORY OF A SINGLE MARKOV OPERATOR
}

\author{
ROBERT SINE
}

\begin{abstract}
A Markov operator acting on the space of continuous functions on a compact Hausdorff space which is uniformly stable in the mean allows a topological ergodic decomposition. A partial converse to this is obtained; if the operator has a decomposition it is then uniformly stable in the mean when restricted to the conservative set. The characterization of uniformly mean stable operators in terms of its invariant structures is the major result. The problem of characterizing the manifolds which can be the invariant manifold for some Markov operator is also considered.
\end{abstract}

1. We denote by $C(X)$ the collection of all continuous real valued functions on the compact Hausdorff space $X$. A Markov operator on $X$ is a bounded linear operator $T$ taking $C(X)$ into $C(X)$ with $T 1=$ $1=\|T\|$. A probability on $X$ will always mean an element $\mu$ in the dual space $C(X)^{*}$ with $\mu(1)=1=\|\mu\|$; the $w^{*}$-compact convex set of all probabilities on $X$ will be denoted by $K$. The following are immediate:

(a) $K$ is invariant under $T^{*}$,

(b) $T$ is order preserving on $C(X)$. The Tychonov fixed point theorem gives

(c) there is a nonempty compact convex set $K_{F}$ of $T^{*}$-invariant probabilities.

We let $M$ be the closed linear manifold of invariant functions; then $M$ contains the constant functions (and possibly nothing more). We obtain a natural decomposition of $X$ from the equivalence relation $x \sim y$ if $f(x)=f(y)$ for all $f$ in $M$. Each set $D$ in this collection $\mathscr{D}$ of sets is closed for it is the intersection of level sets of continuous functions. A partition of a compact Hausdorff space is upper semicontinuous if it is the collection of level sets induced by a continuous map into another compact Hausdorff space [2, p. 132]. If we let $Z$ be the product of the ranges of all of the invariant functions and define $\varphi$ to be the map which sends $x$ into the point of $Z$ with $f(x)$ for its $f^{\text {th }}$ coordinate we see that $\mathscr{D}$ is the level set partition of $\varnothing$ and so is upper semicontinuous. The quotient space $Y=X / \mathscr{D}$ is then compact Hausdorff and the projection map $\pi: X \rightarrow Y$ is continuous and closed. We can lift the map $\pi$ to a map $\Pi: C(Y) \rightarrow C(X)$. The image set of $\Pi$ consists of all continuous functions on $X$ which are constant on each set of $\mathscr{D}$ and $\Pi^{-1} M$ is a subspace of $C(Y)$ which contains 
the constant functions and separates the points of $Y$.

We will call a nonempty closed set $F$ self-supporting if the support of $T^{*} \xi_{x}$ is in $F$ for each $x$ in $F$ ( $\xi_{x}$ is the unit point mass at $x$ and support will always mean the unique closed support). The collection of all self-supporting sets is denoted by $\mathscr{F}$. We have introduced this terminology to avoid the lengthy phrase "topologically and stochastically closed."

THEOREM 1.1. A nonempty closed set $F$ is self-supporting if and only if $T f$ vanishes of $F$ whenever $f$ vanishes on $F$.

Proof. If $F$ is in $\mathscr{F}$ and $f=0$ on $F$ then

$$
T f(x)=\left(f, T^{*} \xi_{x}\right)=\int f d T^{*} \xi_{x}=0 .
$$

On the other hand if $x$ is in $F$ and the support of $T^{*} \xi_{x}$ is not in $F$ we can find a continuous nonnegative function $f$ so that $f=0$ on $F$ and $f$ is strictly positive at some point of the support of $T^{*} \xi_{x}$. Then $0<\int f d T^{*} \xi_{x}=T f(x)$ so $F$ is not in $\mathscr{F}$.

The major importance here of self-supporting sets is that the process can be localized to these sets. For $F$ in $\mathscr{F}$, to define $\left.T\right|_{F}$ on $C(F)$ we let $\left.T\right|_{F} f=\left.T h\right|_{F}$ where $h$ is any continuous extension of $f$ from $F$ to all of $X$. The previous theorem shows that $\left.T\right|_{F}$ is well defined. We now consider specific examples of $\mathscr{F}$-sets.

THEOREM 1.2. Let $f$ be in $M$ and denote the maximizing set of $f$ by $m(f)$. Then $m(f)$ is self-supporting.

Proof. Suppose $\max \{f(x) x \in X\}=r$ and $y$ is in $m(f)$. Then $r=f(y)=T f(y) \leqq T r=r$. Since the $d T^{*} \xi_{y}$ integral of $f$ is the maximum value of $f$, the support of $T^{*} \xi_{y}$ is in the maximizing set.

THEOREM 1.3. Let $\mu$ be an invariant probability. Then $S(\mu)$, the support of $\mu$, is self-supporting.

Proof. If $f=0$ on $S(\mu)$ and $f$ is nonnegative we have

$$
\int T f d \mu=\int f d \mu=0
$$

so we must have for the nonnegative function $T f$ that $T f=0$ on $S(\mu)$. Now if $f$ is any function vanishing on $S(\mu)$ we can look at $f=f^{+}-f^{-}$. 
REMARK. Although the maximizing set of an invariant function is self-supporting the other level sets in general are not. However we do have

THEOREM 1.4. Let $f$ be in $M$ and $\mu$ be an invariant probability with support $S(\mu)$. Then for each closed nonempty set $A$ of reals in the range of $f$ the set $F=f^{-1}(A) \cap S(\mu)$ is self-supporting.

For the proof we need a lemma due to Rosenblatt [10]. If $\mu$ is an invariant probability and $T f=f$ on $S(\mu)$ we say $f$ is $\mu$-invariant.

Lemma 1.5. For each $\mu$ in $K_{F}$, the set of $\mu$-invariant functions forms an algebra.

Proof. It is sufficient to show that $f \vee g$ is $\mu$-invariant whenever both $f$ and $g$ are. Now $f \leqq f \vee g$ and $g \leqq f \vee g$ so $T f \leqq T(f \vee g)$ and $T g \leqq T(f \vee g)$. So $T f \vee T g \leqq T(f \vee g)$ and on $S(\mu)$ we have $f \vee g \leqq T(f \vee g)$. We obtain equality at once on integrating with respect to $\mu$.

Now to prove Theorem 1.4. We restrict $T$ to be a Markov operator on $S(\mu)$. Any $\mu$-invariant function is an invariant function when restricted to $S(\mu)$ so the invariant functions for the restricted operator form an algebra. We can find a continuous function $\varphi$ of a real variable so that $\varphi$ of has $f^{-1}(A) \cap S(\mu)$ as its maximizing set (on $S(\mu)$ ). Now $\varphi$ of is in the algebra since $\varphi \circ f$ is clearly continuous and constant on the sets of the decomposition. Then $f^{-1}(A) \cap S(\mu)$ is a self-supporting set for $T$ on $S(\mu)$; but this is clearly a property that is independent of the embedding of $S(\mu)$ in $X$.

It is standard Zornification that each self-supporting set contains a minimal self-supporting set. Members of the class of minimal selfsupporting sets are disjoint.

THEOREM 1.6. If $F$ is in $\mathscr{F}$ there is an invariant probability with support contained in $F$.

Proof. We can regard $F$ as embedded in $K$ by $x \rightarrow \xi_{x}$. Then $F_{0}=\overline{c o} F$ is compact convex and $T^{*}$-invariant so the result obtains from Tychonov again.

TheoRem 1.7. Let $F$ be a minimal self-supporting set. Then $F$ is the support of an invariant probability and each invariant function is constant on $F$.

Proof. $F$ supports at least one invariant probability; if the sup- 
port is a proper subset of $F$ we contradict minimality. Now if $f$ is invariant the level sets of $f$ restricted to the support can give only a trivial partition of $F$ so $f$ is constant on $F$.

COROLLARY 1.8. Each minimal self-supporting set is contained in exactly one $D$ in $\mathscr{D}$.

Those sets of $\mathscr{D}$ which do support invariant probabilities will be called ergodic sets; the collection of ergodic sets will be devoted by $\mathscr{E}$ and $W=\bigcup\{E: E \in \mathscr{E}\}$ will be called the conservative set.

THEOREM 1.9. The conservative set is closed.

Proof. By properties of upper semicontinuous decompositions if $V_{1}$ is open in $X$ and contains $E$ then there exists an open set $V_{2}$ with $E \subset V_{2} \subset V_{1}$ where $\pi V_{2}$ is a neighborhood of the point $e=\pi E$ in $Y=X / \mathscr{D}$. Now if $x_{\alpha}$ is a net of points in the conservative set $W$ with $x_{\alpha} \rightarrow x_{0}$ we have $\pi x_{\alpha} \rightarrow \pi x_{0}$. For any neighborhood $V_{1}$ of $E$ the ergodic sets $E_{\alpha}$ are ultimately in $V_{2} \subset V_{1}$. Then if we pick $\mu_{\alpha}$ to be any invariant probability on $E_{\alpha}$ we can pass to a subnet so that $\mu_{\alpha} \rightarrow \mu_{0}$ where $\mu_{0}$ is an invariant probability. The support of $\mu_{0}$ is in $E_{0}$ so that $E_{0}$ is an ergodic set.

REMARK. Jamison [3] proves this last result for a uniformly mean stable operator. Lloyd [4] actually works with the Markov projection that always exists for a uniformly mean stable operator; he shows that the set of extremes of $K_{F}$ forms an closed set (in the $w^{*}$ topology). These properties are equivalent for a uniformly mean stable operator. Choquet has given an example of a deterministic operator with a nonclosed extreme point set for $K_{F}$ [8, p. 83].

It is easy to see that the ergodic sets are in general a proper subcollection of $\mathscr{D}$. It is also clear that an ergodic set may contain more than one minimal self-supporting set (consider $T f(x)=f\left(x^{2}\right)$ on $[0,1])$. But it is not at all obvious that a minimal self-supporting set may support more than one invariant probability. Let $N$ be the positive integers; translation $n \rightarrow n+1$ extends to a homeomorphism $\varphi$ of $\beta N \backslash N$ ( $\beta N$ denotes the Stone-Cech compactification). For the reversible deterministic operator $T f=f \circ \varphi$ Alvarez de Araya [1] and Raimi [9] show that each minimal self-supporting set supports at least two distinct invariant probabilities.

THEOREM 1.10. Let $F$ be a minimal self-supporting set and $F_{0}$ be the closed convex hull of the invariant probabilities which are 
supported in $F$. If $\mu$ is an extreme point of $F_{0}$ then $\mu$ is extreme in $K_{F}$.

Proof. Suppose $\mu=(1 / 2)\left(\lambda_{1}+\lambda_{2}\right)$ where $\lambda_{1}$ and $\lambda_{2}$ are in $K_{F}$ and $\mu$ is extreme in $F_{0}$. It is immediate that $\lambda_{1}$ and $\lambda_{2}$ are absolutely $\mu$-continuous and so are supported on $F$. Thus $\mu=\lambda_{1}=\lambda_{2}$.

The converse is false. Again for the example of translation as a homeomorphism on $\beta N \backslash N$ Alvarez de Araya [1, p. 131] asserts that there exist extremes of $K_{F}$ which are not minimally supported.

THEOREM 1.11. Each extreme invariant probability has its support in an ergodic set.

Proof. Let $f$ be an invariant function which is not constant on $S(\mu)$. If we can pick $r$ so that the $\mu$ measure of $[f \leqq r]$ is strictly between zero and one then $[f \leqq r] \cap S(\mu)$ is a self-supporting proper subset of $S(\mu)$. We define a positive invariant measure by $\lambda(A)=$ $\mu(A \cap[f \leqq r])$; then

$$
\mu(A)=\mu[f \leqq r] \frac{\lambda(A)}{\mu[f \leqq r]}+\mu[f>r] \frac{\mu(A \backslash[f \leqq r])}{\mu[f>r]}
$$

shows $\mu$ is not extreme. To show such a choice of $r$ is possible suppose $\mu[f \leqq r]=0$ for all $r<\max \{f(x): x \in S(\mu)\}$. Then the $\mu$ measure of the maximum set of $f$ on $S(\mu)$ is 1 and $f$ is constant on $S(\mu)$. The same argument works as well from the other end and we are done.

The manifold $L=\Pi^{-1} M$ separates the points of $X / \mathscr{D}$ and contains the constant functions. We will call a point $p$ in $X / \mathscr{D}$ a Choquet point (for $L$ ) if the only probability on $X / \mathscr{D}$ which represents the point evaluation functional on $L, f \rightarrow f(p)$, is the point mass $\xi_{p}$. The collection of all Choquet points is called the Choquet boundary for $L$; it is a boundary in the sense that the maximizing set for each function in $L$ meets the Choquet boundary. A very readable guide to Choquet's theorem is Phelps [8]. Although we make no use of the Choquet-Bishop-de Leeuw theorem it is this geometric point of view that we follow throughout. If $D$ is a set in $\mathscr{D}$ and there is a point $x$ in $D$ with the support of $T^{*} \xi_{x}$ not contained in $D$ then $p=\pi D$ is not a Choquet point. Thus the Choquet points of $L$ must be images of ergodic sets in $X$. We give an example in $\S 3$ to show that the image of an ergodic set may fail to be a Choquet point even when the ergodic set is self-supporting.

REMARK. We wish to point out that the self-support property for an ergodic set $E$ is much stronger than the measure theoretic 
result that with probability 1 no mass escapes from $E$ where the probability is any invariant probability supported in $E$. This almost everywhere result follows at once from the fact that the support of every invariant probability is self-supporting.

THEOREM 1.12. $\operatorname{dim} M \leqq \operatorname{dim} K_{F}$ where $\operatorname{dim} i s$ the affine dimension.

Proof. Let $f_{1}, \cdots, f_{n}$ be invariant functions which span an $n$ dimensional subspace $N$. Let $K(N)$ be the positive norm one functionals in $N^{*}$. Then $K(N)$ is clearly $n$-dimensional so has at least $n$ exposed points. Then there are $n$ disjoint sets $F_{1}, \cdots, F_{n}$ in $X$ and $n$ functions $g_{1}, \cdots, g_{n}$ in $N$ so that $g_{i}\left(F_{i}\right)=1$ and $g_{i}\left(X \backslash F_{i}\right)=0$. This gives $n$ disjoint maximizing sets and so $n$ linearly independent invariant probabilities.

2. A Markov operator on $C(X)$ is called uniformal mean stable if the Cesaro means $A_{n} f$ converge uniformly for each $f$ in $C(X)$ (to a necessarily invariant function). The decomposition for such operators has been presented by several authors [3, 4, and 10]. The observation that ergodic sets not only contain self-supporting subsets but are themselves self-supporting appears to be new.

THEOREM 2.1. If $T$ is uniformly mean stable and $f$ is a function constant on each of the sets in $\mathscr{D}$ then there is an invariant function $g$ with $g=f$ on the conservative set.

Proof. The candidate is $\bar{f}=\lim A_{n} f$. We know $\bar{f}$ is constant on each set in $\mathscr{D}$; to see that it is the right constant on each set in $\mathscr{E}$ simply integrate with respect to any invariant probability in $E$.

THEOREM 2.2. If $\mu$ and $\lambda$ are two distinct invariant probabilities for a uniformly mean stable operator then there is an invariant function $f$ which distinguishes in the sense that $(\mu, f)=(\lambda, f)$.

Proof. Pick $f$ to be any function in $C(X)$ which distinguishes $\mu$ and $\lambda$; then $\bar{f}=\lim A_{n} f$ meets the requirements.

Corollary 2.3. If $T$ is uniformly mean stable then there is exactly one minimal self-supporting set in each ergodic set and each minimal self-supporting set is the support of exactly one invariant probability. Moreover the extreme invariant probabilities are the minimally supported invariant probabilities. 
COROLlaRY 2.4. If $T$ is uniformly mean stable each ergodic set is self-supporting.

Proof. Let $B$ be the image of $W$ under $\pi$. It is clear that $\left.L\right|_{B}$ is $C(B)$. Since the Choquet boundary is a subset of $B$ and given any continuous function on $B$ it has an extension to $X / \mathscr{D}$ which is in $L$ we have that $B$ is the Choquet boundary. So each ergodic set is self-supporting.

COROLlaRy 2.5. The conservative set is self-supporting.

Proof. The closure of the union of any collection of self-supporting sets is easily seen to be self-supporting; but $W$ is closed.

Corollary 2.6. If $T$ is uniformly mean stable $\operatorname{dim} M=\operatorname{dim} K_{F}$.

Proof. We need only $\operatorname{dim} M \geqq \operatorname{dim} K_{F}$. Now we can find as many extreme points in $K_{F}$ as its dimension. If there are at least $n$ extremes then there are at least $n$ distinct ergodic sets so at least $n$ points in $\pi W$. Thus $C(\pi W) \cong M$ is at least $n$-dimensional.

We now prove the converse of Theorem 2.2; this is the geometry of the title.

Theorem 2.7. Let $T$ be a Markov operator on $C(X)$. Suppose that the invariant functions separate the invariant probabilities. Then $T$ is uniformly mean stable.

Proof. The $M$ topology of $K_{F}$ defined by $\mu_{\alpha} \rightarrow \mu_{0}[M]$ if and only if $\left(f, \mu_{\alpha}\right) \rightarrow\left(f, \mu_{0}\right)$ for all $f$ in $M$ is Hausdorff by assumption and clearly weaker than the $w^{*}$ topology of $K_{F}$. Thus the two topologies agree. For any point $x$ in $X$ and any $f$ in $M$ we have $\left(T^{n} f, \xi_{x}\right)=$ $\left(f, \xi_{x}\right)=f(x)$ so that the orbit of $T^{*^{n}} \xi_{x}$ has one invariant probability $\mu_{x}$ in its closed convex hull. Then $A_{x}^{*} \xi_{x}$ converges to $\mu_{x}$. We claim that the map $x \rightarrow \mu_{x}=\lim A_{n}^{*} \xi_{x}$ is continuous relative to the $w^{*}$ topology. For if $f$ is in $M$ we have $f\left(x_{\alpha}\right)=\left(f, \mu_{0}\right)$ and if $x_{\alpha} \rightarrow x_{0}$ then $f\left(x_{0}\right)=\left(f, \mu_{0}\right)$ and clearly $f\left(x_{\alpha}\right) \rightarrow f\left(x_{0}\right)$. Now we have an analytic condition known to be equivalent to uniform mean stability [3, Theorem 1.1].

3. We will say that a Markov operator has a topologically ergodic decomposition if each ergodic set is self-supporting and supports exactly one invariant probability. This second condition has the geometric 
interpretation that $M$ distinguishes the extremes of the set of invariant probabilities.

Since each ergodic set is self-supporting we can restrict the action of $T$ to an ergodic set. The restricted operator has a unique invariant probability so on each individual ergodic set $A_{n} f$ converges uniformly to the mean value of $f$ on the set. We can do better than the local uniform convergence on ergodic sets however.

THEOREM 3.1. Let $T$ be a Markov operator with a topological ergodic decomposition. Then the conservative set $W$ is self-supporting and the restricted operator is uniformly mean stable on $W$.

Proof. Clearly $W=\bigcup\{E: E \in \mathscr{C}\}$ is self-supporting. If $f$ is continuous on $W$ and constant on each ergodic set we have $T f=f$ on $W$. If $\lambda$ and $\mu$ are two invariant probabilities which agree on each function in $M$ then $\lambda(A)=\mu(A)$ for each set in the Borel field generated by $\mathscr{D}$. But if $\lambda=\mu$ then there is a continuous function $f$ which distinguishes $\lambda$ and $\mu$ and a Borel function $\bar{f}=\lim A_{n} f$ measurable with respect to the Borel field generated by $\mathscr{D}$ which also distinguishes. Thus the invariant manifold for the restricted operator distinguishes the invariant probabilities so $T$ is uniformly mean stable on $W$ by Theorem 2.7.

We now consider two examples. For the first we take $X$ to be the closed unit disc $\Delta$. For each $x$ in $\Delta$ we define $T^{*} \xi_{x}$ to be the uniformly distributed unit mass on the disc $\Delta_{r}(x)$ centered at $x$ with radius $r$ equal to one half the distance from $\{x\}$ to the set $\partial \Delta \cup\{0\}$. The operator is then defined by $T f(x)=\left(f, T^{*} \xi_{x}\right)$. The invariant functions are easily shown to be the continuous functions on $\Delta$ which are harmonic at each interior point (the origin is a removable singularity). The decomposition $\mathscr{D}$ is a decomposition to points and the ergodic sets are the individual points of $\partial \Delta$ and the point at the origin. So $T$ has a topological ergodic decomposition and is clearly uniformly mean stable when restricted to the conservative set (indeed, the restriction is the identity operator). The origin is a self-supporting ergodic set but its $\pi$-image is not in the Choquet boundary.

The second example is obtained by adding an additional point to the first; let $p$ be a point disjoint from $\Delta$ and set $T^{*} \xi_{p}$ equal to the uniformly distributed unit mass on $\partial \Delta$. Then $f(p)=f(0)$ for each invariant function. The decomposition is the collection of individual points of the deleted disc $\triangle \backslash\{0\}$ together with the two point set $\{0, p\}$. This last set supports the invariant probability $\xi_{0}$ so is ergodic but $T^{*} \xi_{p}$ has support disjoint from $\{0, p\}$ so it is not a self-supporting set.

4. Given a subspace $M$ of $C(X)$ which contains the constant 
functions when is it the invariant manifold of a Markov operator? If we ask that the operator be uniformly mean stable then $M$ is also the invariant manifold of the Markov projection $P=\lim A_{n}$ as well, where the limit is in the strong operator topology. A Markov projection is called smoothing if $P(f P g)=(P f)(P g)$. If $X$ is metrizable there is a partial answer. If the decomposition of $X$ induced by the algebra $A$ is lower semicontinuous (so that $\pi: X \rightarrow X / \mathscr{D}$ is open as well as closed and continuous) then Lloyd [5] observes that a selection theorem of Michael [7] gives the existence of a Markov smoothing projection onto $A$. To show the lower semicontinuity condition is not necessary Lloyd gives a smoothing Markov projection on the algebra of functions continuous on $[0,2]$ and constant on $[0,1]$. But some additional restriction is necessary for Lloyd also gives the following example where it is easily shown that there is no Markov projection on the algebra (smoothing is free if the range is an algebra). $A$ is the space of functions continuous on $[0,3]$ which are constant on $[1,2]$.

Before we can state our results we need some terminology. If $L$ is a subspace of $C(X)$ which separates points and contains the constant functions we will say that $L$ is a simplex if the Choquet boundary is a closed set and each point evaluation functional has a unique representing probability supported on the Choquet boundary. Our terminology is not standard here but we can avoid delicate measuretheoretic interpretations of a probability supported by the boundary.

REMARK. Since the Choquet boundary is assumed closed it is uniqueness that is the essential factor in the definition. For a point evaluation functional will extend to the space of all continuous functions on the boundary and the Riesz theorem gives a representation as a probability on the boundary.

We will call a simplex $L$ a Markov simplex if $L$ when restricted to $\partial L$ is $C(\partial L)$.

THEOREM 4.1. If $M$ is a separating subspace of $C(X)$ containing constants then there is a Markov projection onto $M$ if and only if $M$ is a Markov simplex.

Proof. Suppose $M$ is the invariant manifold of a Markov projection $P$. The Choquet boundary is closed as a projection is always uniformly mean stable. If $f$ is any function continuous on the Choquet boundary we can extend $f$ continuously to all of $X$ and then take the Cesaro limit to obtain an invariant function which agrees with $f$ on the boundary. Uniqueness then follows from the uniqueness assertion in the Riesz theorem. On the other hand suppose $L$ is a simplex. Given 
$x$ in $X$ we wish to define $P$ so that $P^{*} \xi_{x}=\mu_{x}$ where $\mu_{x}$ is the unique representing probability for $x$. We need only show that $P$ acts continuously. It is known that the map $\mu_{x} \rightarrow x$ is $w^{*}$ continuous [8, p. 4]. The uniqueness gives the existence of the inverse map and so $x \rightarrow \mu_{x}$ is a homeomorphism by elementary topology.

If $M$ fails to separate points of $X$ we cannot do nearly as well.

THEOREM 4.2. (a) If $M$ is the invariant manifold for a Markov projection then $L=\Pi^{-1} M$ is a Markov simplex in $C(X / \mathscr{D})$.

(b) Let $A$ be the algebra generated by a manifold $M$ which contains the constants. If $X$ is metrizable and if the decomposition generated by $A$ is lower semicontinuous then $M$ is the range of a Markov projection if and only if $L=\Pi^{-1} M$ is a Markov simplex in $C(X / \mathscr{D})$.

Proof. (a) By uniform mean stability again the closed set $\pi W$ is the Choquet boundary for $L$ and we again get both existence and uniqueness for representing measures from the Hahn-Banach and Riesz theorems.

(b) The Lloyd-Michael result gives a Markov smoothing projection onto $A$. This followed by the projection $P: C(X / \mathscr{D}) \rightarrow L$ of Theorem 4.1 with proper indentifications gives one direction. On the other hand if a projection exists we can restrict it to $A \cong C(X / \mathscr{D})$ and use the reverse implication of Theorem 4.1.

We give an example to show that Markov projections may exist without factoring through the enveloping algebra. Let $M$ be the manifold of functions continuous on $[0,3]$ which are linear on both $[0,1]$ and $[2,3]$ and constant on $[1,2]$. If we define $P^{*}$ by

$$
\begin{array}{ll}
P^{*} \mathscr{O}_{x}=(1-x) \xi_{0}+x \lambda & 0 \leqq x \leqq 1 \\
P^{*} \xi_{x}=\lambda & 1<x<2 \\
P^{*} \mathscr{f}_{x}=(x-2) \lambda+(3-x) \xi_{3} & 2 \leqq x \leqq 3
\end{array}
$$

where $\lambda$ is Lebesgue measure on $[1,2]$ we obtain a Markov projection onto $M$. Now the algebra generated by $M$ is the functions continuous on $[0,3]$ and constant on [1,2]. This is Lloyd's example of an algebra with no Markov projection.

From Theorem 4.1 we see that there is no Markov projection on the manifold spanned by $1, x$ and $x^{2}$ in the space of continuous function on $[0,1]$. We can extend this negative result with a theorem suggested by an approximation theorem of Bohrman and Korovkin [6, p. 7].

REMARK. This approximation theorem has an elementary proof 
but while the hypotheses are easy to meet for applications (the Bernstein and the Fejer approximation theorems) they are difficult to interpret. More objectionable from our point of view the hypotheses force the compact Hausdorff space to be a subset of a finite dimensional Euclidean space.

THEOREM 4.3. Let $\left\{T_{n}\right\}$ be a sequence of positive operators and $L$ a subspace of $C(X)$ which separates points and contains the constant functions.

(a) If for each $f$ in $L$ we have $T_{n} f^{2}$ converging to $f^{2}$ with the convergence bounded and pointwise then $T_{n}$ converges to the identity operator in the weak operator topology.

(b) If the convergence of $T_{n} f^{2}$ to $f^{2}$ is uniform then $T_{n}$ converges to the identity operator in the strong operator topology.

Proof. We have $T_{n} 1$ converging to 1 where the convergence is bounded and pointwise so that the norms $\left\|T_{n}\right\|$ are uniformly bounded. For any $x$ and any $y=x$ we can pick $f$ in $L$ with $f(x)=0$ and $f(y)=0$. Then $\left(T_{n} f^{2}\right)(x)=\left(f^{2}, T_{n}^{*} \xi_{x}\right)$ and if $\mu$ is any (necessarily positive) measure in the cluster set of $T_{n}^{*} \xi_{x}$ we have $y$ disjoint from the support of $\mu$. Since $\mu(1)=1$ and $S(\mu)$ is $\{x\}$ we have $\mu=\xi_{x}$. Then $T_{n}^{*} \xi_{x}$ converges to $\xi_{x}$ in the $w^{*}$-topology which proves (a).

Now suppose convergence is not in the strong operator topology. Then for some subsequence of $\left\{T_{n}\right\}$ and some $g$ in $C(X)$ we have $\left\|T_{n} g-g\right\|>r>0$. For the subsequence we can pick $x_{n}$ in $X$ to get $\left|T_{n} g\left(x_{n}\right)-g\left(x_{n}\right)\right|>r>0$. By passing to a subnet of the subsequence we can assume $x_{\alpha} \rightarrow x$. Let $\mu$ be any $w^{*}$ cluster point of $T_{\alpha}^{*} \xi\left(x_{\alpha}\right)$. Then for $f$ in $L$ with $f\left(x_{0}\right)=0$ we have that $T_{\alpha} f^{2}\left(x_{\alpha}\right)$ clusters at $\int f^{2} d \mu$. Since $T_{\alpha} f^{2}$ converges uniformly to $f^{2}$ we have

$$
\int f^{2} d \mu=f^{2}\left(x_{0}\right)=[0
$$

and so as before $\mu=\xi\left(x_{0}\right)$. Finally

$$
\left|T_{\alpha} g\left(x_{\alpha}\right)-g\left(x_{\alpha}\right)\right| \leqq\left|T_{\alpha} g\left(x_{\alpha}\right)-g\left(x_{0}\right)\right|+\left|g\left(x_{0}\right)-g\left(x_{\alpha}\right)\right|
$$

gives the contradiction as both terms go to zero.

REMARK. Convergence of the $T_{n}$ on the squares of the "coordinate functions" of $X$ cannot be replaced with convergence on the "coordinate functions" themselves. Let $T_{n}$ be the second and third degree Bernstein operators alternately.

COROLlary 4.4. Let $M$ be a subspace of $C(X)$ which contains 
the constants and separates points. If $T$ is a Markov operator which leaves the square of each function in $M$ invariant then $T$ is the identity operator.

\section{BIBLIOGRAPHY}

1. J. Alvarez de Araya, Invariant measures on compactifications of the integers, Thesis, University of Washington, 1963.

2. J. G. Hocking and G. S. Young, Topology, Addison-Wesley, Reading, 1961.

3. B. Jamison, Ergodic decompositions induced by certain Markov operators, Trans. Amer. Math. Soc. 117 (1965), 451-468.

4. S. P. Lloyd, On certain projections in spaces of continuous functions, Pacific J. Math. 13 (1963), 171-175.

5. - On extreme averaging operators, Proc. Amer. Math. Soc. 14 (1963), 305310.

6. G. G. Lorentz, Approximation of Functions, Holt, Rinehart, and Winston, New York, 1966.

7. E. Michael, A linear mapping between function spaces, Proc. Amer. Math. Soc. 15 (1964), 407-409.

8. R. R. Phelps, Lectures on Choquent's Theorem, Van Nostrand, Princeton, 1966.

9. R. A. Raimi, Minimal sets and ergodic measures in $\beta N \backslash N$, Bull. Amer. Math. Soc. 70 (1964), 711-712.

10. M. Rosenblatt, Equicontinuous Markov operators, Teor. Veronjatnost. i Primenen. $\mathbf{9}$ (1964), 205-222.

Received August 11, 1967. This researth was partially supported by the National Science Foundation.

UNIVERSITY OF MINNESOTA 


\section{PACIFIC JOURNAL OF MATHEMATICS}

EDITORS

H. ROYDEN

Stanford University

Stanford, California

R. R. Phelps

University of Washington

Seattle, Washington 98105
J. DugundJI

Department of Mathematics

University of Southern California

Los Angeles, California 90007

\section{RICHARD ARENS}

University of California

Los Angeles, California 90024

\section{ASSOCIATE EDITORS}
E. F. BeCKENBACH
B. H. NeUmanN
F. WOLF
K. YOSIDA

\section{SUPPORTING INSTITUTIONS}

UNIVERSITY OF BRITISH COLUMBIA CALIFORNIA INSTITUTE OF TECHNOLOGY UNIVERSITY OF CALIFORNIA MONTANA STATE UNIVERSITY UNIVERSITY OF NEVADA NEW MEXICO STATE UNIVERSITY OREGON STATE UNIVERSITY UNIVERSITY OF OREGON OSAKA UNIVERSITY UNIVERSITY OF SOUTHERN CALIFORNIA
STANFORD UNIVERSITY UNIVERSITY OF TOKYO UNIVERSITY OF UTAH WASHINGTON STATE UNIVERSITY UNIVERSITY OF WASHINGTON

AMERICAN MATHEMATICAL SOCIETY CHEVRON RESEARCH CORPORATION TRW SYSTEMS NAVAL WEAPONS CENTER 


\section{Pacific Journal of Mathematics \\ Vol. 27, No. $1 \quad$ January, 1968}

Willard Ellis Baxter, On rings with proper involution ............... 1

Donald John Charles Bures, Tensor products of $W^{*}$-algebras........... 13

James Calvert, Integral inequalities involving second order derivatives . . . . 39

Edward Dewey Davis, Further remarks on ideals of the principal class.... 49

Le Baron O. Ferguson, Uniform approximation by polynomials with integral

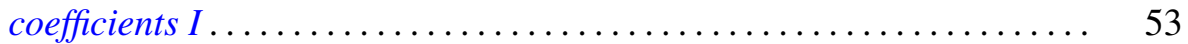

Francis James Flanigan, Algebraic geography: Varieties of structure

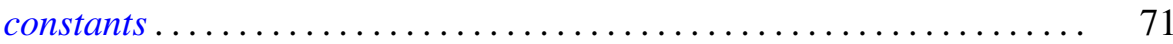

Denis Ragan Floyd, On QF -1 algebras ..................... 81

David Scott Geiger, Closed systems of functions and predicates ......... 95

Delma Joseph Hebert, Jr. and Howard E. Lacey, On supports of regular Borel measures ................................... 101

Martin Edward Price, On the variation of the Bernstein polynomials of a function of unbounded variation ........................ 119

Louise Arakelian Raphael, On a characterization of infinite complex matrices mapping the space of analytic sequences into itself........ 123

Louis Jackson Ratliff, Jr., A characterization of analytically unramified

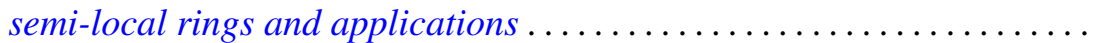

S. A. E. Sherif, A Tauberian relation between the Borel and the Lototsky transforms of series ................................ 145

Robert C. Sine, Geometric theory of a single Markov operator .......... 155

Armond E. Spencer, Maximal nonnormal chains in finite groups......... 167

Li Pi Su, Algebraic properties of certain rings of continuous functions .... 175

G. P. Szegô, A theorem of Rolle's type in $E^{n}$ for functions of the class $C^{1} \ldots 193$

Giovanni Viglino, A co-topological application to minimal spaces ........ 197

B. R. Wenner, Dimension on boundaries of $\varepsilon$-spheres ............... 201 